UDC 530.1, 538.0; PACS 05.20.-y, 05.60.-a, 05.70.Ln

\title{
GENERALIZED HYDRODYNAMICS OF MULTI-COMPONENT FLUIDS
}

\author{
I.M.MRYGLOD \\ Institute for Condensed Matter Physics \\ of the Ukrainian National Academy of Sciences, \\ 1 Svientsitskii St., UA-290011 Lviv, Ukraine
}

Received February 17, 1997

\begin{abstract}
Starting from the rigorous microscopic approach and taking into account all the conserved quantities of multi-component, chemically nonreactive fluids, the basic equations of generalized hydrodynamics are derived. The explicit expressions for the transport coefficients as well as the damping coefficients and dispersions of collective hydrodynamic modes are found. It is shown that these results are in agreement with those obtained by phenomenological theories.
\end{abstract}

\section{Introduction}

During the last decade an essential progress in understanding of dynamical properties of fluids has been achieved in connection with the method of the generalized collective modes. The concept of generalized collective modes proposed initially in Ref. $[1,2]$ for the study of time correlation functions of dense one-component fluids, represents now a modern and powerful method, which allows to obtain the self-consistent description of dynamical properties within wide range of wave-numbers and frequencies starting from the hydrodynamic values and up to the Gaussian-like range. In this framework, time correlation functions could be written as a weighted sum of partial terms, each of them being associated with the relevant generalized collective mode could be expressed via the corresponding eigenvector and eigenvalue of so-called generalized operator of evolution. Some of the generalized collective modes correspond in the hydrodynamic limit to the well-known hydrodynamic excitations. The other ones are called the kinetic modes and have higher eigenvalues with finite damping coefficients in the hydrodynamic limit. It is important to note that this approach is based on the extended set of dynamic variables containing, in addition to the conserved variables, their higher-order time derivatives.

In [3] the parameter-free generalized collective modes approach, based on Markovian approximation for the higher order memory functions, has been suggested. In this paper, the five- and seven-variable descriptions of longitudinal fluctuations for a pure Lennard-Jones liquid has been developed. The extension of the formalism to a nine-variable description has been presented in $[4,5]$. The calculations of time correlation functions for a Lennard-Jones fluid [6] showed that in a wide range of $k$ and $\omega$ a reasonable agreement of the theory with MD data can be already observed within lower-order mode approximations.

Very promising results have been aslo obtained for binary mixtures. The theoretical and experimental investigations showed that the dynamic

(C) I.M.Mryglod, 1997

ISSN 0452-9910. Condensed Matter Physics 1997 No 10 (115-135) 115 
structure factors $S(k, \omega)$ of liquid water [7], liquid $\mathrm{Li}_{4} \mathrm{~Pb}$ [8] and of dense gas mixtures He-Ne [9] as well as He-Ar [10,11] display a behavior, which can be explained only in terms of new generalized collective modes, namely, so-called fast sound.

Until recently, the theoretical description of the dynamics for multicomponent fluids was based to a great extent on phenomenological approaches $[12,13]$ and on the methods of kinetic theory (see, e.g., [14,15]) for low density gas mixtures. The main goal of this paper is: to derive the basic equations of generalized hydrodynamics for multi-component, chemically nonreactive fluids; to study these equations in the hydrodynamic limit of small wave-numbers $k$ and frequencies $\omega$; to analyse the spectrum of the hydrodynamic modes. Considering these problems one may create the basis for the next study of multi-component fluids within the generalized mode approach.

The structure of the paper is following: section 2 outlines the main initial relations which are used in the paper; in section 3 we present the basic equations of the generalized hydrodynamics for a general mixture; the results for static correlation functions in the hydrodynamic limit are derived in section 4 by means of thermodynamic fluctuation theory; in sections 5 and 6 we present the calculations for the elements of hydrodynamic frequency and memory functions matrixes; the study of the hydrodynamic collective modes for a mixture is performed in Section 7; we end with a discussion in section 8 .

\section{Initial relations}

We consider for a multi-component fluid an open region of volume $V$ containing $N_{1}, N_{2}, \ldots, N_{\nu}$ particles of species $1,2, \ldots, \nu$. Hence, $N=\sum_{\alpha=1}^{\nu} N_{\alpha}$ is a total number of particles.

Let us introduce the following notations

$$
n_{\alpha}=\frac{N_{\alpha}}{V}
$$

is the number density of particles in the $\alpha$-th species,

$$
n=\sum_{\alpha=1}^{\nu} n_{\alpha}=\frac{N}{V}
$$

is the total number density of particles,

$$
\rho_{\alpha}=\frac{m_{\alpha} N_{\alpha}}{V}=m_{\alpha} n_{\alpha}=m_{\alpha} c_{\alpha} n
$$

is the mass density of particles in the $\alpha$-th species,

$$
\rho=\sum_{\alpha=1}^{\nu} \rho_{\alpha}=\frac{M}{V}=\bar{m} n
$$

is the total mass density of particles, where $M=\sum_{\alpha=1}^{\nu} m_{\alpha} N_{\alpha}$ and $\bar{m}=$ $M / N=\sum_{\alpha=1}^{\nu} m_{\alpha} c_{\alpha}$,

$$
c_{\alpha}=\frac{N_{\alpha}}{N}=\frac{n_{\alpha}}{n}
$$


is the concentration of the $\alpha$-th particles in mixture $\left(\sum_{\alpha=1}^{\nu} c_{\alpha}=1\right)$, and

$$
x_{\alpha}=\frac{M_{\alpha}}{M}=\frac{\rho_{\alpha}}{\rho}=\frac{m_{\alpha}}{\bar{m}} c_{\alpha}
$$

is the mass concentration of the $\alpha$-th particles in mixture $\left(\sum_{\alpha=1}^{\nu} x_{\alpha}=1\right)$.

For mixtures the partial structure factors are defined as follows [24,25]

$$
\begin{array}{r}
S_{\alpha \gamma}(k)=\delta_{\alpha \gamma}+\left(n_{\alpha} n_{\gamma}\right)^{1 / 2} \int \mathrm{d} \mathbf{r} \mathbf{r}^{2}\left(g_{\alpha \gamma}(r)-1\right) \mathrm{e}^{i \mathbf{r k}} \\
=\delta_{\alpha \gamma}+4 \pi\left(n_{\alpha} n_{\gamma}\right)^{1 / 2} \int_{0}^{\infty} \mathrm{d} r r^{2}\left(g_{\alpha \gamma}(r)-1\right) \frac{\sin k r}{k r}
\end{array}
$$

where $g_{\alpha \gamma}(r)$ is the partial (two-particle) radial distribution function which is given by

$$
g_{\alpha \gamma}(r)=\frac{1}{n_{\alpha} n_{\gamma}}\left\langle\left(\sum_{i, j=1}^{N_{\alpha}, N_{\gamma}}\right)^{\prime} \frac{1}{V} \delta\left(\mathbf{r}-\mathbf{R}_{i}^{\alpha}-\mathbf{R}_{j}^{\gamma}\right)\right\rangle .
$$

On occasion the Faber-Ziman partial structure factors are also used $[24,25]$

$$
\begin{gathered}
a_{\alpha \gamma}(k)=1+n \int d \mathbf{r} \mathbf{r}^{2}\left(g_{\alpha \gamma}(r)-1\right) \mathrm{e}^{i \mathbf{r k}} \\
=1+4 \pi n \int_{0}^{\infty} d r r^{2}\left(g_{\alpha \gamma}(r)-1\right) \frac{\sin k r}{k r}
\end{gathered}
$$

It immediately follows from (7) and (9) that

$$
S_{\alpha \gamma}(k)=\delta_{\alpha \gamma}+\left(c_{\alpha} c_{\gamma}\right)^{1 / 2}\left(a_{\alpha \gamma}(r)-1\right) .
$$

Note that in the limit $k \rightarrow \infty$ one has

$$
S_{\alpha \gamma}(k) \rightarrow \delta_{\alpha \gamma}, \text { and } a_{\alpha \gamma}(k) \rightarrow 1 .
$$

The total static structure factor could be defined as

$$
S_{\mathrm{NN}}(k)=\sum_{\alpha, \gamma=1}^{\nu}\left(c_{\alpha} c_{\gamma}\right)^{1 / 2} S_{\alpha \gamma}(k)=\sum_{\alpha, \gamma=1}^{\nu} c_{\alpha} c_{\gamma} a_{\alpha \gamma}(k) .
$$

The microscopic set of the conserved (hydrodynamic) variables may be introduced in the form

$$
\hat{P}_{\mathbf{k}}^{h}=\left\{\hat{\mathcal{N}}_{\mathbf{k}}, \hat{\mathbf{J}}_{\mathbf{k}}, \hat{E}_{\mathbf{k}}\right\}
$$

where

$$
\hat{\mathcal{N}}_{\mathbf{k}}=\left\{\hat{N}_{\mathbf{k}, \alpha}\right\}
$$


is a column-vector with components $\hat{N}_{\mathbf{k}, \alpha}$,

$$
\hat{N}_{\mathbf{k}, \alpha}=\sum_{i=1}^{N_{\alpha}} \exp \left\{i \mathbf{k} \mathbf{R}_{i}^{\alpha}\right\}
$$

is the number density of particles in the $\alpha$-th species;

$$
\hat{\mathbf{J}}_{\mathbf{k}}=\sum_{i=1}^{N_{\alpha}} \hat{\mathbf{J}}_{\mathbf{k}, \alpha}
$$

is the density of the total current, $\hat{\mathbf{J}}_{\mathbf{k}}=\left\{\hat{J}_{\mathbf{k}}^{x}, \hat{J}_{\mathbf{k}}^{y}, \hat{J}_{\mathbf{k}}^{z}\right\}$ and

$$
\hat{\mathbf{J}}_{\mathbf{k}, \alpha}=\sum_{i=1}^{N_{\alpha}} \mathbf{p}_{i}^{\alpha} \exp \left\{i \mathbf{k R}_{i}^{\alpha}\right\}=\sum_{i=1}^{N_{\alpha}} m_{\alpha} \mathbf{v}_{i}^{\alpha} \exp \left\{i \mathbf{k R}_{i}^{\alpha}\right\},
$$

are the current densities of particles in the $\alpha$-th species;

$$
\hat{E}_{\mathbf{k}}=\sum_{\alpha=1}^{\nu} \hat{E}_{\mathbf{k}, \alpha}=\sum_{\alpha=1}^{\nu} \sum_{i=1}^{N_{\alpha}} e_{i}^{\alpha} \exp \left\{i \mathbf{k} \mathbf{R}_{i}^{\alpha}\right\},
$$

is the total energy density, where

$$
e_{i}^{\alpha}=\frac{\left(\mathbf{p}_{i}^{\alpha}\right)^{2}}{2 m_{\alpha}}+\frac{1}{2} \sum_{\gamma=1}^{\nu}\left(\sum_{j=1}^{N_{\gamma}}\right)^{\prime} V_{\alpha \gamma}\left(\left|\mathbf{R}_{i}^{\alpha}-\mathbf{R}_{j}^{\gamma}\right|\right)
$$

and $V_{\alpha \gamma}\left(\left|\mathbf{r}-\mathbf{r}^{\prime}\right|\right)$ is a potential of interparticle interactions, and the symbol $\left({ }^{\prime}\right)$ in (18) indicates that $i \neq j$ when $\gamma=\alpha$.

For all the dynamic variables introduced above the microscopic equations of motion could be written in the form

$$
\stackrel{\circ}{P_{\mathbf{k}, l}^{h}}-i \mathbf{k} I_{\mathbf{k}, l}^{h}=0
$$

where $\stackrel{\circ}{P_{\mathbf{k}, l}^{h}} \equiv i L_{N} \hat{P}_{\mathbf{k}, l}^{h}$ and $i L_{N}$ is a Liouville operator. The equation (19) is in fact the conservation law in the local form. The quantities $I_{\mathbf{k}, l}^{h}$ are the (microscopic) hydrodynamic fluxes.

Turning our attention to the conserved dynamic variables, we should in principle consider a $(\nu+3+1)$-component set containing $\nu$-component of the number density $\hat{N}_{\mathbf{k}, \alpha}$ of particles in each species, the three components of the total current density $\hat{\mathbf{J}}_{\mathbf{k}}$, and the total energy density $\hat{E}_{\mathbf{k}}$. However, from the microscopic equations of motion (19) it follows that the number densities $\hat{N}_{\mathbf{k}, \alpha}$ and the energy density $\hat{E}_{\mathbf{k}}$ are coupled only with the longitudinal component of $\hat{\mathbf{J}}_{\mathbf{k}}$, directed along $k$. This is because of space isotropy of the system. Thus, we have the same situation as in the case of simple liquids. As a result, one may split the set of the hydrodynamic variables into two separate subsets. The first set $\hat{P}_{\mathbf{k}}^{h, l}$ includes the variables $\left\{\hat{\mathcal{N}}_{\mathbf{k}}, \hat{J}_{\mathbf{k}}^{l}, \hat{E}_{\mathbf{k}}\right\}$ and may be called as the set of longitudinal hydrodynamic variables, and 
the second one $\hat{P}_{\mathbf{k}}^{h, t}$ comprises two components of the total current perpendicular to $\mathbf{k}$ (transverse currents). It is evident that the final equations for two transverse components should be the same because of isotropy of the system. Therefore, one may suppose that the vector $\mathbf{k}$ is directed along $0 Z$ axis, so that we have finally the $(\nu+2)$-component longitudinal set $\hat{P}_{\mathbf{k}}^{h, l} \equiv\left\{\hat{\mathcal{N}}_{k}, \hat{J}_{k}^{z}, \hat{E}_{k}\right\}$ and the two component transverse set $\hat{P}_{\mathbf{k}}^{h, t} \equiv\left\{\hat{J}_{k}^{x}, \hat{J}_{k}^{y}\right\}$ of dynamic variables describing multi-component fluids.

\section{Generalized hydrodynamics of mixtures}

\subsection{General framework}

It has been shown $[16,17]$ that the macroscopic equations of motion for an arbitrary set of the dynamic variables

$$
\hat{P}_{k}=\left\{\hat{P}_{k, 1}, \hat{P}_{k, 2}, \ldots, \hat{P}_{k, \mathrm{~L}}\right\},
$$

where $\hat{P}_{k}$ is a column-vector, could be written in the matrix form as follows

$$
\left\{i \omega I^{\mathrm{L} \times \mathrm{L}}-i \Omega^{\mathrm{L} \times \mathrm{L}}+\tilde{\varphi}_{\epsilon}^{\mathrm{L} \times \mathrm{L}}(\omega)\right\}\langle\Delta \hat{P}\rangle^{\omega}=0
$$

where $I^{\mathrm{L} \times \mathrm{L}}$ is the $\mathrm{L} \times \mathrm{L}$ unit matrix and $\Delta \hat{P}_{k, i}=\hat{P}_{k, i}-\left\langle\hat{P}_{k, i}\right\rangle$,

$$
i \Omega^{\mathrm{L} \times \mathrm{L}}=\left(\stackrel{\circ}{P}, \hat{P}^{+}\right)\left(\hat{P}, \hat{P}^{+}\right)^{-1}
$$

is a so-called frequency matrix $\left(\stackrel{\circ}{P} \equiv i L_{N} \hat{P}\right)$, and

$$
\begin{aligned}
& \tilde{\varphi}_{\epsilon}^{\mathrm{L} \times \mathrm{L}}(\omega) \equiv \tilde{\varphi}^{\mathrm{L} \times \mathrm{L}}(\epsilon+i \omega)= \\
& =\left((1-\mathcal{P}) \stackrel{\circ}{P}, \frac{1}{i \omega+\epsilon+(1-\mathcal{P}) i \hat{L}_{N}}(1-\mathcal{P}) \stackrel{\circ}{P}^{+}\right)\left(\hat{P}, \hat{P}^{+}\right)^{-1}
\end{aligned}
$$

is a matrix of the memory functions. $\hat{P}^{+}$in (22) and (23) is a transposed vector with the elements $\left\{\hat{P}_{-k, i}\right\}$. The Mori-like projection operator $\mathcal{P}$ is defined by the relation

$$
\mathcal{P} \ldots=\mathcal{P}_{\mathrm{L}} \ldots=\left(\ldots, \hat{P}^{+}\right)\left(\hat{P}, \hat{P}^{+}\right)^{-1} \hat{P} .
$$

In equations (22), (23), and (24) for the static correlation functions we used the notation

$$
(\hat{A}, \hat{B})=\langle\Delta \hat{A} \Delta \hat{B}\rangle,
$$

where $\langle\ldots\rangle$ denotes the expectation value.

The matrix equation for the Laplace transform $\tilde{\mathcal{F}}^{\mathrm{L} \times \mathrm{L}}(k, z)$ of the equilibrium time correlation functions $\mathcal{F}^{\mathrm{L} \times \mathrm{L}}(k, t)$, where

$$
\begin{gathered}
\mathcal{F}^{\mathrm{L} \times \mathrm{L}}(k, t)=\left\langle\Delta \hat{P}_{k} \mathrm{e}^{-i L_{N} t} \Delta \hat{P}_{-k}^{+}\right\rangle, \\
\tilde{\mathcal{F}}^{\mathrm{L} \times \mathrm{L}}(k, z)=\int_{0}^{\infty} \mathrm{d} t \exp \{-z t\} \mathcal{F}^{\mathrm{L} \times \mathrm{L}}(k, t),
\end{gathered}
$$


has the similar structure to (21), namely,

$$
\left\{z I^{\mathrm{L} \times \mathrm{L}}-i \Omega^{\mathrm{L} \times \mathrm{L}}+\tilde{\varphi}^{\mathrm{L} \times \mathrm{L}}(z)\right\} \tilde{\mathcal{F}}^{\mathrm{L} \times \mathrm{L}}(k, z)=\mathcal{F}^{\mathrm{L} \times \mathrm{L}}(k, 0) .
$$

The matrix of memory functions could be also written in the another form

$$
\begin{aligned}
& \tilde{\varphi}^{\mathrm{L} \times \mathrm{L}}(z)=\left(\left(\stackrel{\circ}{P}, \stackrel{\circ}{P^{+}}\right)^{z}-\right. \\
& \left.-\left(\stackrel{\circ}{P}, \Delta \hat{P}^{+}\right)^{z} \frac{1}{\left(\Delta \hat{P}, \Delta \hat{P}^{+}\right)^{z}}\left(\Delta \hat{P}, \stackrel{\circ}{P^{+}}\right)^{z}\right) \frac{1}{\left(\Delta \hat{P}, \Delta \hat{P}^{+}\right)},
\end{aligned}
$$

which may be useful for some applications.

It is worth noting that the time correlation functions $\mathcal{F}^{\mathrm{L} \times \mathrm{L}}(k, t)$ are closely related to the classical retarded correlation Green functions

$$
G_{A B}^{(r)}\left(t-t^{\prime}\right)=-i \theta\left(t-t^{\prime}\right)\left\langle A(t) B\left(t^{\prime}\right)\right\rangle
$$

where $\theta(t)=1$ or 0 according to whether $t>0$ or $t>0$, so that as it follows from (28) the spectrum of collective modes to be found from the equation

$$
\text { Det }\left|z I^{\mathrm{L} \times \mathrm{L}}-i \Omega^{\mathrm{L} \times \mathrm{L}}+\tilde{\varphi}^{\mathrm{L} \times \mathrm{L}}(z)\right|=0
$$

gives the poles of the Green functions constructed on the set of dynamic variables $\left\{\Delta \hat{P}_{k, i}\right\}$. It should be also stressed that the matrix equation for the equilibrium time correlation functions (28) is in fact the exact equation until the explicit expressions for the frequency and memory function matrixes are used. This statement can be proved using the expressions for the frequency matrix (22) and the matrix of memory functions (29).

Note that as it follows from (22), (23), and (24) it is convenient for concrete calculations to use the set orthogonalized dynamic variables possessing the properties

$$
\left\langle\Delta \hat{P}_{k, i} \Delta \hat{P}_{-k, j}\right\rangle=\delta_{i j}\left\langle\Delta \hat{P}_{k, j} \Delta \hat{P}_{-k, j}\right\rangle,
$$

so that one has

$$
\left(\hat{P}_{k}, \hat{P}_{k}^{+}\right)_{j i}^{-1}=\delta_{j i}\left(\hat{P}_{k, i}, \hat{P}_{-k, i}\right)^{-1} .
$$

The linearized transport equations (21), the equations for the equilibrium time correlation functions (28), and the equation for collective mode spectrum (31) form a general basis for the study of the dynamic behavior of fluids in the memory function formalism.

We note that in the expressions given above the sum over wave-vector $\mathbf{k}$ has to be performed together with a summation of the index $i$ of the dynamic variables.

\subsection{Frequency matrix and matrix of memory functions for a multi-component fluid}

Let us apply the results presented above for the set of dynamic variables which contains the conserved variables of a multi-component fluid $\hat{P}_{\mathbf{k}}^{h}$. By analogy with a case of simple fluid, for longitudinal components we will start from the set of dynamic variables

$$
\hat{P}_{k}^{l}=\left\{\hat{\mathcal{N}}_{k}, \hat{J}_{k}, \hat{H}_{k}\right\},
$$


where

$$
\hat{H}_{k}=\hat{E}_{k}-\left(\hat{E}_{k}, \hat{\mathcal{N}}_{k}^{+}\right)\left(\hat{\mathcal{N}}_{k}, \hat{\mathcal{N}}_{k}^{+}\right) \hat{\mathcal{N}}_{k}
$$

is so-called the enthalpy density, and

$$
\mathcal{P}_{\mathcal{N}} \ldots=\left(\ldots, \mathcal{N}^{+}\right)\left(\hat{\mathcal{N}}, \hat{\mathcal{N}}^{+}\right)^{-1} \hat{\mathcal{N}}=\sum_{\alpha \gamma}\left(\ldots, \hat{N}_{\alpha}\right)\left(\hat{\mathcal{N}}, \hat{\mathcal{N}}^{+}\right)_{\alpha \gamma}^{-1} \hat{N}_{\gamma}
$$

is Mori-like projection operator. We note that in contrary to the case of simple fluids the hydrodynamic set (33) is not orthogonal in sense of (32) because of $\left(\hat{N}_{k, \alpha}, \hat{N}_{-k, \beta}\right) \neq 0$ for $\alpha \neq \beta$.

Using the properties of correlation functions under time inversion and spatial symmetry operations, one can show that in general the structure of the $(\nu+2) \times(\nu+2)$ hydrodynamic frequency matrix is following

$$
i \Omega^{H}(k)=\left(\begin{array}{ccc}
0^{\nu \times \nu} & i \Omega_{\mathrm{NJ}}^{\nu \times 1}(k) & 0^{\nu \times 1} \\
i \Omega_{\mathrm{JN}}^{1 \times \nu}(k) & 0 & i \Omega_{\mathrm{JH}} \\
0^{1 \times \nu} & i \Omega_{\mathrm{HJ}} & 0
\end{array}\right),
$$

where the notation $0^{\alpha \times \beta}$ denotes the $\alpha \times \beta$ matrix, all the elements of which are zero.

For arbitrary $k$ all the elements of the memory functions matrix are not equal to zero, so that

$$
\varphi^{H}(k, t)=\left(\begin{array}{ccc}
\varphi_{\mathrm{NN}}^{\nu \times \nu}(k, t) & \varphi_{\mathrm{NJ}}^{\nu \times 1}(k, t) & \varphi_{\mathrm{NH}}^{\nu \times 1}(k, t) \\
\varphi_{\mathrm{JN}}^{1 \times \nu}(k, t) & \varphi_{\mathrm{JJ}}(k, t) & \varphi_{\mathrm{JH}}(k, t) \\
\varphi_{\mathrm{HN}}^{1 \times \nu}(k, t) & \varphi_{\mathrm{HJ}}(k, t) & \varphi_{\mathrm{HH}}(k, t)
\end{array}\right) .
$$

Using the equations (21), (28) and (31) for the hydrodynamic set of dynamic variables $\hat{P}_{k}^{l}$ we obtain the basis equations of generalized hydrodynamics for longitudinal components of a multi-component fluids. The generalized transport coefficients of the system can be introduced in usual way [17] via the elements of matrix $\tilde{\varphi}^{H}(k, z)$.

For the transverse fluctuations with one variable hydrodynamic set

$$
\hat{P}_{k}^{t}=\hat{J}_{k}^{t},
$$

one has the generalized transport equation in the form

$$
\left\{i \omega+\tilde{\varphi}_{\mathrm{JJ}}^{t}(k, \omega+i \varepsilon)\right\}\left\langle\Delta \hat{J}_{k}^{t}\right\rangle^{\omega}=0,
$$

which is quite similar as it is for a simple fluid. The generalized shear viscosity can be defined via the memory function $\tilde{\varphi}_{\mathrm{JJ}}^{t}(k, z)$.

\section{Static correlation functions and thermodynamic theory of fluctuations}

The space and time response of the system near the equilibrium state may be calculated using: (i) the thermodynamic fluctuation theory to provide the initial values for the static correlation functions as well as (ii) the linearized 
hydrodynamic equations to determine the modes by which the system returns to equilibrium.

Let us consider the expressions for the static correlation functions

$$
F(k)=\left(\hat{P}_{k}^{h}, \hat{P}_{-k}^{h}\right)
$$

in the limit $k \rightarrow 0$ and discuss the thermodynamic relations for these functions which follow from the theory of thermodynamic fluctuations. We start with the equilibrium Gibbs distribution $\hat{\rho}$ for the grand canonical ensemble $\left[\left(V, T,\left\{\mu_{\alpha}\right\}\right)\right.$-ensemble $]$

$$
\rho_{\lambda, N}=\exp \left[\beta\left(\Omega-E_{\lambda, N}+\sum_{\alpha=1}^{\nu} \mu_{\alpha} N_{\alpha}\right)\right],
$$

where $\Omega=\Omega\left(V, T,\left\{\mu_{\alpha}\right\}\right)=-P V$ is the thermodynamic potential depending on temperature $k_{\mathrm{B}} T=1 / \beta$, chemical potentials $\mu_{\alpha}$ of the $\alpha$-th species. $V$ is fixed and $P$ indicates the pressure. The index " $\lambda$ " in (40) labels the value of $E_{N}$. The potential is defined as usual by the condition that $\hat{\rho}$ is normalized to unity.

For an arbitrary operator $\hat{a}$ the following equalities are easy to prove

$$
\begin{aligned}
\frac{\partial\langle\hat{a}\rangle}{\partial \mu_{\alpha}} & =\beta\left(\hat{a}, \hat{N}_{\alpha}\right), \\
\frac{\partial\langle\hat{a}\rangle}{\partial \beta} & =-(\hat{a}, \hat{w}),
\end{aligned}
$$

where $\hat{w}=\hat{E}-\sum_{\alpha=1}^{\nu} \mu_{\alpha} \hat{N}_{\alpha}$, and the quantities $\hat{N}_{\alpha}$ and $\hat{E}$ are the values of the dynamic variables $\hat{N}_{k, \alpha}$ and $\hat{E}_{k}$ in the limit $k \rightarrow 0$, respectively.

As a result in the grand canonical ensemble we have

$$
\frac{\partial\left\langle\hat{N}_{\alpha}\right\rangle}{\partial \mu_{\gamma}}=\frac{\partial\left\langle\hat{N}_{\gamma}\right\rangle}{\partial \mu_{\alpha}}=\beta\left(\hat{N}_{\alpha}, \hat{N}_{\gamma}\right)=\beta\left(\hat{\mathcal{N}}, \hat{\mathcal{N}}^{+}\right)_{\alpha \gamma}
$$

Note that

$$
\left(\hat{N}_{k, \alpha}, \hat{N}_{-k, \gamma}\right) \equiv\left(\hat{\mathcal{N}}_{k}, \hat{\mathcal{N}}_{k}^{+}\right)_{\alpha \gamma}=N\left(c_{\alpha} c_{\gamma}\right)^{1 / 2} S_{\alpha \gamma}(k) .
$$

As it follows from (43)

$$
\left(\frac{\partial \mu_{\alpha}}{\partial N_{\gamma}}\right)_{T, V, N_{\bar{\gamma}}}=\frac{1}{\beta}\left(\hat{\mathcal{N}} \hat{\mathcal{N}}^{+}\right)_{\alpha \gamma}^{-1}
$$

where the index $\bar{\gamma}$ denotes all species different from $\gamma$.

To make a contact with experimental quantities we now transform the thermodynamic derivatives at constant volume into derivatives at constant pressure, by means of the identity

$$
\left(\frac{\partial \mu_{\alpha}}{\partial N_{\gamma}}\right)_{T, V, N_{\bar{\gamma}}}=\left(\frac{\partial \mu_{\alpha}}{\partial N_{\gamma}}\right)_{T, P, N_{\bar{\gamma}}}+\frac{v_{\alpha} v_{\gamma}}{V \kappa_{\mathrm{T}}}
$$


where

$$
v_{\gamma}=\left(\frac{\partial V}{\partial N_{\gamma}}\right)_{T, P, N_{\bar{\gamma}}}
$$

is the partial molar volume, per molecule, of species $\gamma$, and

$$
\kappa_{\mathrm{T}}=-\frac{1}{V}\left(\frac{\partial V}{\partial P}\right)_{T, N_{\gamma}}
$$

is the isothermal compressibility.

By making use of Gibbs-Duhem equation

$$
S \mathrm{~d} T-V \mathrm{~d} P+\sum_{\alpha=1}^{\nu} N_{\alpha} \mathrm{d} \mu_{\alpha}=0
$$

we find for isothermal-isobaric processes that

$$
\sum_{\alpha=1}^{\nu}\left\langle N_{\alpha}\right\rangle\left(\frac{\partial \mu_{\alpha}}{\partial N_{\gamma}}\right)_{T, P, N_{\bar{\gamma}}}=0
$$

Using this equation and taking into account that

$$
\sum_{\alpha=1}^{\nu} c_{\alpha} v_{\alpha}=v=\frac{V}{N}
$$

one gets the equation for $v_{\gamma}$

$$
\frac{v_{\gamma}}{\kappa_{\mathrm{T}}}=\sum_{\alpha=1}^{\nu}\left\langle N_{\alpha}\right\rangle\left(\frac{\partial \mu_{\alpha}}{\partial N_{\gamma}}\right)_{T, V, N_{\bar{\gamma}}}=\frac{1}{\beta} \sum_{\alpha=1}^{\nu}\left\langle N_{\alpha}\right\rangle\left(\hat{\mathcal{N}} \hat{\mathcal{N}}^{+}\right)_{\alpha \gamma}^{-1}
$$

and the equation for $\kappa_{\mathrm{T}}$

$$
\begin{aligned}
\frac{1}{\kappa_{\mathrm{T}}}=\frac{1}{V} \sum_{\alpha, \gamma=1}^{\nu}\left\langle N_{\alpha}\right\rangle\left\langle N_{\gamma}\right\rangle\left(\frac{\partial \mu_{\alpha}}{\partial N_{\gamma}}\right)_{T, V, N_{\gamma}}= & \frac{1}{\beta V} \sum_{\alpha, \gamma=1}^{\nu}\left\langle N_{\alpha}\right\rangle\left(\hat{\mathcal{N}} \hat{\mathcal{N}}^{+}\right)_{\alpha \gamma}^{-1}\left\langle N_{\gamma}\right\rangle \\
& =N \frac{n}{\beta} \sum_{\alpha, \gamma=1}^{\nu} c_{\alpha}\left(\hat{\mathcal{N}} \hat{\mathcal{N}}^{+}\right)_{\alpha \gamma}^{-1} c_{\gamma} .
\end{aligned}
$$

The last equation can be used for the definition of the generalized compressibility $\kappa_{\mathrm{T}}(k)$

$$
\frac{1}{\kappa_{\mathrm{T}}(k)}=N n k_{\mathrm{B}} T \sum_{\alpha, \gamma=1}^{\nu} c_{\alpha}\left(\hat{\mathcal{N}}_{k}, \hat{\mathcal{N}}_{k}^{+}\right)_{\alpha \gamma}^{-1} c_{\gamma}
$$

Using the thermodynamic relation

$$
\left(\frac{\partial E}{\partial \beta}\right)_{V, N_{\alpha}}=\left(\frac{\partial E}{\partial \beta}\right)_{V, \mu_{\alpha}}-\sum_{\alpha \gamma}\left(\frac{\partial E}{\partial \mu_{\alpha}}\right)_{T, V, \mu_{\bar{\alpha}}}\left(\frac{\partial \mu_{\alpha}}{\partial N_{\gamma}}\right)_{T, V, N_{\bar{\gamma}}}\left(\frac{\partial N_{\gamma}}{\partial \beta}\right)_{V, \mu_{\alpha}}
$$


and recalling (41), (42) one finds

$$
\begin{array}{r}
\left(\frac{\partial E}{\partial \beta}\right)_{V, N_{\alpha}}=-(\hat{E}, \hat{w})+\sum_{\alpha \gamma}\left(\hat{E}, \hat{N}_{\alpha}\right)\left(\hat{\mathcal{N}}, \hat{\mathcal{N}}^{+}\right)_{\alpha \gamma}^{-1}\left(\hat{N}_{\gamma}, \hat{w}\right) \\
=-\left(\hat{E},\left(1-\mathcal{P}_{\mathcal{N}}\right) \hat{w}\right)
\end{array}
$$

where $\mathcal{P}_{\mathcal{N}}$ is is given by (35), so that $\mathcal{P}_{\mathcal{N}} \hat{N}_{\alpha}=\hat{N}_{\alpha}$. Hence, one has

$$
\begin{aligned}
\left(\frac{\partial E}{\partial \beta}\right)_{V, N_{\alpha}}=- & \left.\left(\left(1-\mathcal{P}_{\mathcal{N}}\right) \hat{E},\left(1-\mathcal{P}_{\mathcal{N}}\right) \hat{w}\right)\right)= \\
& \left.-\left(\left(1-\mathcal{P}_{\mathcal{N}}\right) \hat{E},\left(1-\mathcal{P}_{\mathcal{N}}\right) \hat{E}\right)\right)=-(\hat{H}, \hat{H}),
\end{aligned}
$$

where $\hat{H}$ is the value of the dynamic variables $\hat{H}_{k}$ when $k \rightarrow 0$. To derive (56) we have used the equality

$$
((1-\mathcal{P}) A, B)=(A,(1-\mathcal{P}) B)=((1-\mathcal{P}) A,(1-\mathcal{P}) B)
$$

which holds for an arbitrary Mori-like projection operator $\mathcal{P}$.

Finally, we get

$$
k_{\mathrm{B}} T^{2} C_{\mathrm{V}}=-\left(\frac{\partial E}{\partial \beta}\right)_{V, N_{\alpha}}=(\hat{H}, \hat{H})
$$

or in the more general form

$$
k_{\mathrm{B}} T^{2} C_{\mathrm{V}}(k)=\left(\hat{H}_{k}, \hat{H}_{-k}\right) .
$$

The equation (58) is in fact the definition of the generalized specific heat at constant volume in the grand canonical ensemble. Note that the orthogonal variables $\hat{J}_{k}$ and $\hat{N}_{k, \alpha}$ are orthogonal in sense of the equation (20) to $\hat{H}_{k}$.

For the calculations of the frequency matrix we need also to know the static correlation function $\left(\stackrel{\circ}{J}_{k}, \hat{h}_{-k}\right)$ in the limit $k \rightarrow 0$. Taking into account the relation

$$
\stackrel{\circ}{J}_{k}=i k \sigma^{z z}(k),
$$

where $\sigma^{z z}(k)$ is the $z z$-component of stress tensor, one can find from (42) $\left(\sigma^{z z}=\sigma^{z z}(0)\right)$

$$
\left(\frac{\partial\left\langle\sigma^{z z}\right\rangle}{\partial \beta}\right)_{V, \mu_{\alpha}}=-\left(\sigma^{z z}, \hat{w}\right)+\frac{1}{\beta} \sum_{\alpha=1}^{\nu} \mu_{\alpha}\left(\frac{\partial\left\langle\sigma^{z z}\right\rangle}{\partial \mu_{\alpha}}\right)_{T, V, \mu_{\bar{\alpha}}}
$$

or

$$
\left(\sigma^{z z}, \hat{E}\right)=\frac{1}{\beta}\left[T S+\sum_{\alpha=1}^{\nu} \mu_{\alpha}\left\langle N_{\alpha}\right\rangle\right]
$$

where we used the equality

$$
\left\langle\sigma^{z z}\right\rangle=P V=-\Omega .
$$


As a result one gets

$$
\begin{aligned}
\left(\sigma^{z z}, \hat{H}\right) & =\left(\sigma^{z z}, \hat{E}\right)-\sum_{\alpha, \gamma}\left(\frac{\partial\langle\hat{E}\rangle}{\partial \mu_{\alpha}}\right)_{T, V, \mu_{\bar{\alpha}}}\left(\frac{\partial \mu_{\alpha}}{\partial N_{\gamma}}\right)_{T, V, N_{\bar{\gamma}}}\left\langle N_{\gamma}\right\rangle \\
= & \frac{T}{\beta}\left[S-\sum_{\alpha=1}^{\nu} N_{\alpha}\left(\frac{\partial S}{\partial N_{\alpha}}\right)_{T, V, N_{\bar{\alpha}}}\right]=\frac{T V}{\beta}\left(\frac{\partial P}{\partial T}\right)_{V, N_{\alpha}},
\end{aligned}
$$

or

$$
(\stackrel{\circ}{J}, \hat{H})=i k \frac{T V}{\beta}\left(\frac{\partial P}{\partial T}\right)_{V, N_{\alpha}} .
$$

In order to prove the equality

$$
S-\sum_{\alpha=1}^{\nu} N_{\alpha}\left(\frac{\partial S}{\partial N_{\alpha}}\right)_{T, V, N_{\bar{\alpha}}}=V\left(\frac{\partial P}{\partial T}\right)_{V, N_{\alpha}}
$$

one may consider the free energy $F$,

$$
\mathrm{d} F=-S \mathrm{~d} T-P \mathrm{~d} V+\sum_{\alpha=1}^{\nu} \mu_{\alpha} \mathrm{d} N_{\alpha}
$$

Introducing the normalized quantity $f=F / V, N_{\alpha}=\rho_{\alpha} V, s=S / N$, one finds

$$
f=-P+\sum_{\alpha=1}^{\nu} \mu_{\alpha} \rho_{\alpha}
$$

and

$$
\mathrm{d} f=-s \mathrm{~d} T-P \mathrm{~d} V+\sum_{\alpha=1}^{\nu} \mu_{\alpha} \mathrm{d} \rho_{\alpha}
$$

Hence, we have

$$
\left(\frac{\partial f}{\partial T}\right)_{\rho_{\alpha}}=-s=-\left(\frac{\partial P}{\partial T}\right)_{\rho_{\alpha}}+\sum_{\alpha=1}^{\nu} \rho_{\alpha}\left(\frac{\partial \mu_{\alpha}}{\partial T}\right)_{\rho_{\alpha}} .
$$

By making use now Maxwell relation

$$
\left(\frac{\partial \mu_{\alpha}}{\partial T}\right)_{\rho_{\alpha}}=-\left(\frac{\partial s}{\partial \rho_{\alpha}}\right)_{T, \rho_{\bar{\alpha}}},
$$

we get finally

$$
\left(\frac{\partial P}{\partial T}\right)_{\rho_{\alpha}}=s-\sum_{\alpha=1}^{\nu} \rho_{\alpha}\left(\frac{\partial s}{\partial \rho_{\alpha}}\right)_{T, \rho_{\bar{\alpha}}}=\frac{1}{V}\left[S-\sum_{\alpha=1}^{\nu} N_{\alpha}\left(\frac{\partial S}{\partial N_{\alpha}}\right)_{T, N_{\bar{\alpha}}}\right] .
$$




\section{Hydrodynamic frequency matrix}

Using the expressions (44), (53), (58), and (62) we can calculate the matrix elements of $i \Omega^{H}(k)$. All the other needful static correlation functions are more simple for calculations. For example, the flux of the variable $\hat{N}_{k, \alpha}$ is

$$
\stackrel{\circ}{N}_{k, \alpha}=\frac{i k}{m_{\alpha}} \hat{J}_{k, \alpha}
$$

so that

$$
\left(\stackrel{\circ}{N}_{k, \alpha}, \hat{J}_{-k}\right)=\left(\stackrel{\circ}{J}_{k}, \hat{N}_{-k, \alpha}\right)=\frac{i k}{\beta} N_{\alpha}=\frac{i k}{\beta} N c_{\alpha}
$$

and

$$
\left(\hat{J}_{-k}, \hat{J}_{-k}\right)=\sum_{\alpha=1}^{\nu} N_{\alpha} \frac{m_{\alpha}}{\beta}=N \frac{\bar{m}}{\beta} .
$$

Hence, for the elements $i \Omega_{\mathrm{NJ}}^{\alpha}(k)$ and $i \Omega_{\mathrm{JN}}^{\alpha}(k)$ of the frequency matrix one has

$$
\begin{array}{r}
i \Omega_{\mathrm{NJ}}^{\alpha}(k)=\left({\stackrel{\circ}{N_{k, \alpha}}} \hat{J}_{-k}\right)\left(\hat{J}_{k}, \hat{J}_{-k}\right)^{-1}=\frac{i k}{\bar{m}} c_{\alpha}, \\
i \Omega_{\mathrm{JN}}^{\alpha}(k)=\sum_{\gamma=1}^{\nu}\left(\stackrel{\circ}{J}_{k}, \hat{N}_{-k, \gamma}\right)\left(\hat{\mathcal{N}}_{k} \hat{\mathcal{N}}_{k}^{+}\right)_{\gamma \alpha}^{-1} \\
=\frac{i k}{\beta} \sum_{\gamma=1}^{\nu} N_{\gamma}\left(\hat{\mathcal{N}}_{k} \hat{\mathcal{N}}_{k}^{+}\right)_{\gamma \alpha}^{-1}=i k \frac{v_{\alpha}(k)}{\kappa_{\mathrm{T}}(k)},
\end{array}
$$

where we used (51) and $v_{\alpha}(k)$ may be considered as the $k$-dependent partial molar volume of species $\gamma$ (see also (53)).

Taking into account that

$$
\left(\frac{\partial P}{\partial T}\right)_{V, N_{\alpha}}=-\left(\frac{\partial P}{\partial V}\right)_{T, N_{\alpha}}\left(\frac{\partial V}{\partial T}\right)_{P, N_{\alpha}}=\frac{\alpha_{\mathrm{P}}}{\kappa_{\mathrm{T}}}
$$

where

$$
\alpha_{\mathrm{P}}=\frac{1}{V}\left(\frac{\partial V}{\partial T}\right)_{P, N_{\alpha}}
$$

is the linear thermal expansion coefficient, and introducing the notation for the generalized linear thermal expansion coefficient in the form

$$
\alpha_{\mathrm{P}}(k)=\frac{1}{i k}\left(\stackrel{\circ}{J}_{k}, \hat{H}_{-k}\right) \frac{\kappa_{\mathrm{T}}(k)}{k_{\mathrm{B}} T^{2} V},
$$

we get

$$
\begin{gathered}
i \Omega_{\mathrm{JH}}(k)=\left(\stackrel{\circ}{J}_{k}, \hat{H}_{-k}\right)\left(\hat{H}_{k} \hat{H}_{-k}\right)^{-1}=i k \frac{\alpha_{\mathrm{P}}(k) V}{\kappa_{\mathrm{T}}(k) C_{\mathrm{V}}(k)}, \\
i \Omega_{\mathrm{HJ}}(k)=\left(\stackrel{\circ}{J}_{k}, \hat{h}_{-k}\right)\left(\hat{J}_{k} \hat{J}_{-k}\right)^{-1}=i k \frac{\alpha_{\mathrm{P}}(k) T}{n \bar{m} \kappa_{\mathrm{T}}(k)} .
\end{gathered}
$$


Thus, the hydrodynamic frequency matrix can be written in quite general form as follows

$$
i \Omega^{H}(k)=\left(\begin{array}{ccc}
0^{\nu \times \nu} & \frac{i k}{\bar{m}} \mathbf{c} & 0^{\nu \times 1} \\
i k \frac{\mathbf{v}^{+}(k)}{\kappa_{\mathrm{T}}(k)} & 0 & i k \frac{\alpha_{\mathrm{P}}(k) V}{\kappa_{\mathrm{T}}(k) C_{\mathrm{V}}(k)} \\
0^{1 \times \nu} & i k \frac{\alpha_{\mathrm{P}}(k) T}{\rho \kappa_{\mathrm{T}}(k)} & 0
\end{array}\right) .
$$

where $\mathbf{c}$ and $\mathbf{v}$ are column-vectors with the components $c_{\alpha}$ and $v_{\alpha}(k)$, respectively. In the hydrodynamic limit from (72) one gets

$$
i \Omega^{H}=\left.i \Omega^{H}(k)\right|_{k \rightarrow 0}=\left(\begin{array}{ccc}
0^{\nu \times \nu} & \frac{i k}{\bar{m}} \mathbf{c} & 0^{\nu \times 1} \\
\frac{i k}{\kappa_{\mathrm{T}}} \mathbf{v}^{+} & 0 & i k \frac{V}{C_{\mathrm{v}}} \frac{\alpha_{\mathrm{P}}}{\kappa_{\mathrm{T}}} \\
0^{1 \times \nu} & i k \frac{T}{\rho} \frac{\alpha_{\mathrm{P}}}{\kappa_{\mathrm{T}}} & 0
\end{array}\right) .
$$

Using the result (73) we can study the ideal hydrodynamics (without dissipations) of a many-component fluid and calculate the dispersion of sound waves for the system.

To find the dispersion of sound waves we have to solve the equation

$$
\operatorname{det}\left|z I^{(\nu+2) \times(\nu+2)}-i \Omega^{H}\right|=0 .
$$

Taking into account the structure of the matrix $i \Omega^{H}$, the last equation can be written in the form

$$
z^{\nu}\left[z^{2}-i \Omega_{\mathrm{JH}} i \Omega_{\mathrm{HJ}}-\sum_{\alpha=1}^{\nu} i \Omega_{\mathrm{JN}}^{\alpha} i \Omega_{\mathrm{NJ}}^{\alpha}\right]=0 .
$$

Hence, non-trivial solutions can be found from the equation

$$
z^{2}+k^{2} \frac{T}{\rho \mathrm{C}_{\mathrm{V}}} \frac{\alpha_{\mathrm{P}}^{2}}{\kappa_{\mathrm{T}}^{2}}+k^{2} \frac{\mathbf{v}^{+} \mathbf{c}}{\kappa_{T} \bar{m}}=0,
$$

where $\mathrm{C}_{\mathrm{v}}=C_{\mathrm{v}} / V$. Let us consider the terms proportional to $k^{2}$ in (75) separately, taking into account that

$$
\mathbf{v}^{+} \mathbf{c} \equiv v=\frac{V}{N}=\frac{1}{n}
$$

and $n \bar{m}=\rho$. Therefore, one has

$$
k^{2} \frac{\mathbf{v}^{+} \mathbf{c}}{\kappa_{T} \bar{m}}=k^{2} \frac{1}{\kappa_{T} \rho} .
$$


From the other side, using the well-known relation

$$
\mathrm{C}_{\mathrm{P}}=\mathrm{C}_{\mathrm{V}}+T \frac{\alpha_{\mathrm{P}}^{2}}{\kappa_{\mathrm{T}}}
$$

we get

$$
k^{2} \frac{T}{\rho \mathrm{C}_{\mathrm{V}}} \frac{\alpha_{\mathrm{P}}^{2}}{\kappa_{\mathrm{T}}^{2}}=k^{2} \frac{1}{\rho \kappa_{T}} \frac{\mathrm{C}_{\mathrm{P}}-\mathrm{C}_{\mathrm{V}}}{\mathrm{C}_{\mathrm{V}}}=k^{2} \frac{\gamma-1}{\rho \kappa_{T}}
$$

where

$$
\gamma=\frac{\mathrm{C}_{\mathrm{P}}}{\mathrm{C}_{\mathrm{V}}}
$$

is the ratio of the specific heats at constant pressure and constant volume. Note that the generalized specific heat at constant pressure $\mathrm{C}_{\mathrm{P}}(k)$ and the generalized ratio of the specific heats $\gamma(k)$ can be defined, using the expressions for $\mathrm{C}_{\mathrm{V}}(k), \kappa_{\mathrm{T}}(k)$ and $\alpha_{\mathrm{P}}(k)$ given above. Thereafter, from the equation

$$
z^{2}+k^{2} \frac{\gamma}{\rho \kappa_{\mathrm{T}}}=0
$$

the expected results for the sound velocity immediately follows

$$
c_{s}^{2}=\frac{\gamma}{\rho \kappa_{\mathrm{T}}}
$$

We note also that the elements of the hydrodynamic frequency matrix for the transverse components of current are equal to zero as it should be.

\section{Hydrodynamic matrix of memory functions}

The memory functions are defined on the basis of generalized fluxes

$$
\begin{gathered}
i k I_{k, \alpha}^{d}=\left(1-\mathcal{P}_{H}\right) i L_{N} \hat{N}_{k, \alpha}=\frac{i k}{m_{\alpha}}\left(\hat{J}_{k, \alpha}-\frac{m_{\alpha} c_{\alpha}}{\bar{m}} \hat{J}_{k}\right), \\
i k I_{k, \mathrm{~J}}^{d}=\left(1-\mathcal{P}_{H}\right) i L_{N} \hat{J}_{k}=i k \sigma^{z z}(k)-\sum_{\alpha=1}^{\nu} i \Omega_{\mathrm{JN}}^{\alpha}(k) \hat{N}_{k, \alpha}-i \Omega_{\mathrm{JH}}(k) \hat{H}_{k}, \\
i k I_{k, \mathrm{H}}^{d}=\left(1-\mathcal{P}_{H}\right) i L_{N} \hat{h}_{k}=i k I_{k, \mathrm{H}}-i \Omega_{\mathrm{HJ}}(k) \hat{J}_{k},
\end{gathered}
$$

where the explicit expression for $I_{k, \mathrm{H}}$ follows from the definition

$$
i k I_{k, \mathrm{H}}=i L_{N} \hat{H}_{k}=i k Q(k)-\sum_{\alpha \beta}\left(\hat{E}_{k}, \hat{N}_{-k, \alpha}\right)\left(\hat{\mathcal{N}}_{k}, \hat{\mathcal{N}}_{k}^{+}\right)_{\alpha \beta}^{-1} \hat{J}_{k, \beta}
$$

and $Q^{z}(k) \equiv i L_{N} \hat{E}_{k} / i k$ is the flux of energy.

Thereafter the Laplace transforms of the hydrodynamic memory functions can be written via the generalized kinetic coefficients $L_{l g}(k, z)$ as follows

$$
\tilde{\varphi}_{l l^{\prime}}(\mathbf{k}, z)=k^{2} V k_{\mathrm{B}} T \sum_{g} \tilde{L}_{l g}(k, z)\left(\hat{P}_{k}^{h}, \hat{P}_{-k}^{h}\right)_{g l^{\prime}}^{-1}
$$


where $l, l^{\prime}, g=\{\alpha, \mathrm{J}, \mathrm{H}$,$\} and \alpha=1,2, \ldots, \nu$. We have introduced the generalized kinetic coefficients $\tilde{L}_{l g}(k, z)$ as the Laplace transforms of timedependent functions $L_{l g}(k, t)$ defined on the set of the generalized fluxes $I_{k, l}^{d}$,

$$
L_{l g}(k, t)=\frac{\beta}{V}\left(I_{k, l}^{d}, \exp \left\{-\left(1-\mathcal{P}_{H}\right) i L_{N} t\right\} I_{-k, l}^{d}\right) .
$$

Let us consider now in more detail the hydrodynamic limit $k \rightarrow 0$. In such a case one gets for the fluxes

$$
\begin{gathered}
\left.I_{k, \mathrm{~J}}^{d}\right|_{k \rightarrow 0}=\sigma^{z z}-\frac{1}{\kappa_{T}} \sum_{\alpha=1}^{\nu} v_{\alpha} \hat{N}_{k, \alpha}-\frac{1}{\mathrm{C}_{\mathrm{V}}} \frac{\alpha_{\mathrm{P}}}{k_{\mathrm{T}}} \hat{H}_{k}=I_{\mathrm{J}}^{d}, \\
\left.I_{k, \mathrm{H}}^{d}\right|_{k \rightarrow 0}=I_{\mathrm{H}}-\frac{T}{\rho} \frac{\alpha_{\mathrm{P}}}{k_{\mathrm{T}}} \hat{J}_{k}=I_{\mathrm{H}}^{d},
\end{gathered}
$$

where

$$
\begin{array}{r}
I_{\mathrm{H}}=Q^{z}(0)-\sum_{\alpha=1}^{\nu}\left(\frac{\partial E}{\partial N_{\alpha}}\right)_{T, V, N_{\bar{\alpha}}} \frac{1}{m_{\alpha}} \hat{J}_{k, \alpha} \\
=\left[Q^{z}-\frac{1}{\bar{m}} \hat{J}_{k} \sum_{\alpha=1}^{\nu}\left(\frac{\partial E}{\partial N_{\alpha}}\right)_{T, V, N_{\bar{\alpha}}}\right]-\sum_{\alpha=1}^{\nu}\left(\frac{\partial E}{\partial N_{\alpha}}\right)_{T, V, N_{\bar{\alpha}}} J_{k, \alpha}^{d} .
\end{array}
$$

Note that

$$
\sum_{\alpha=1}^{\nu} m_{\alpha} J_{k, \alpha}^{d}=\sum_{\alpha=1}^{\nu}\left(\hat{J}_{k, \alpha}-\frac{m_{\alpha} c_{\alpha}}{\bar{m}} \hat{J}_{k}\right) \equiv 0 .
$$

The property (87), as it will be seen below, is very important from the view-point of the properties of transport coefficients describing diffusivity.

Let us consider the leading terms in wave vector $k$ of the memory functions (83) when $k$ is small. In such a case the Markovian approximation is valid. Hence, we may neglect the elements $\varphi_{\mathrm{JH}}, \varphi_{\mathrm{JN}}^{1 \times \alpha}$, and $\varphi_{\mathrm{HJ}}, \varphi_{\mathrm{NJ}}^{\alpha \times 1}$ in (37) because they contribute to the collective mode spectrum in the next order of $k$ (the static correlation functions $\left(i L_{N} \hat{J}, i L_{N} \hat{h}\right)$ and $\left(i L_{N} \hat{J}, i L_{N} \hat{N}_{\alpha}\right)$ are equal to zero as follows from the symmetry reasons). Then all other terms in the matrix (37) are proportional to $k^{2}$.

A further simplification can be reached by observing that the equality

$$
\left.\left(I_{k, l}^{d}, \exp \left\{-\left(1-\mathcal{P}_{H}\right) i L_{N} t\right\} I_{-k, l^{\prime}}^{d}\right)\right|_{k \rightarrow 0}=\left(\Delta I_{l}^{d}, \mathrm{e}^{-i L_{N} t} \Delta I_{l^{\prime}}^{d}\right)
$$

could be used in the limit $k \rightarrow 0$, where $I_{l}^{d}$ are the fluxes defined by the equations (79)-(81) when $k=0$. Thus, in the Markovian approximation for the hydrodynamic memory functions we can express the Laplace transforms of the functions (88) via the kinetic coefficients $L_{l j}$ which are defined in usual way as

$$
L_{l l^{\prime}}=\frac{\beta}{V} \int_{0}^{\infty} \mathrm{d} t\left(\Delta I_{l}^{d}, \mathrm{e}^{-i \hat{L}_{N} t} \Delta I_{l^{\prime}}^{d}\right) .
$$

It is seen in (89) that the kinetic coefficients $L_{l l^{\prime}}$ are given via Green-Kubo like formulas. 
The symmetry properties of the transport coefficients immediately follow from the symmetry properties of the generalized $I_{l}^{d}$ fluxes under time reversal transformation,

$$
L_{l l^{\prime}}=L_{l^{\prime} l}
$$

for any non-zero $L_{l l^{\prime}}$ and $l, l^{\prime}$ are from the set $\{\alpha, \mathrm{J}, \mathrm{H}\}$ where $\alpha=1,2, \ldots, \nu$.

The kinetic coefficients $L_{l l^{\prime}}$ can be considered as the well-known transport coefficients, namely:

a) $L_{\mathrm{JJ}}=\eta_{l}=\frac{4}{3} \eta+\zeta$ is the longitudinal viscosity, where $\eta$ and $\zeta$ are the bulk and shear viscosities, respectively;

b) $L_{\mathrm{Hн}}$ defines the thermal conductivity $\lambda\left(L_{h h}=T \lambda\right)^{1}$;

c) $L_{\mathrm{NN}}^{\alpha \beta} \equiv D_{\alpha \beta}$ are the diffusion coefficients;

d) $L_{\mathrm{NH}}^{\alpha} \equiv K_{\alpha}$ describes thermal diffusion in the $\alpha$-th species.

For the transverse components of current we found

$$
L_{\mathrm{JJ}}^{(t)}=\frac{\beta}{V} \int_{0}^{\infty} \mathrm{d} t\left(\sigma^{z x}, \mathrm{e}^{-i \hat{L} t} \sigma^{z x}\right)=\eta,
$$

where $\eta$ is the shear viscosity and $\sigma^{z x}$ is a nondiagonal element of the stress tensor.

The diffusion $D_{\alpha \beta}$ and thermal diffusion $K_{\alpha}$ coefficients possess the additional properties which immediately follow from (87),

$$
\sum_{\alpha=1}^{\nu} m_{\alpha} D_{\alpha \beta}=\sum_{\beta=1}^{\nu} D_{\alpha \beta} m_{\beta} \equiv 0
$$

and

$$
\sum_{\alpha=1}^{\nu} m_{\alpha} K_{\alpha} \equiv 0
$$

Hence, we are now in position to present the matrix of memory functions in the hydrodynamic limit. This matrix is frequency independent and only contains terms of order $k^{2}$

$$
\left.\tilde{\varphi}(k, 0)\right|_{k \rightarrow 0}=\left(\begin{array}{ccc}
k^{2} \phi_{\mathrm{NN}}^{\alpha \beta} & 0 & k^{2} \phi_{\mathrm{NH}}^{\alpha \times 1} \\
0 & k^{2} \phi_{\mathrm{JJ}} & 0 \\
k^{2} \phi_{\mathrm{HN}}^{1 \times \alpha} & 0 & k^{2} \phi_{h h}
\end{array}\right),
$$

where

$$
\begin{array}{cc}
\phi_{\mathrm{NN}}^{\alpha \delta}=V \sum_{\gamma=1}^{\nu} D_{\alpha \gamma}\left(\frac{\partial \mu_{\delta}}{\partial N_{\gamma}}\right)_{T, V, N_{\bar{\gamma}}}=\sum_{\gamma=1}^{\nu} D_{\alpha \gamma}\left(\frac{\partial \mu_{\delta}}{\partial n_{\gamma}}\right)_{T, n_{\bar{\gamma}}}, \\
\phi_{\mathrm{NH}}^{\alpha \times 1}=\frac{1}{T \mathrm{C}_{\mathrm{V}}} K_{\alpha}, & \phi_{\mathrm{HN}}^{1 \times \alpha}=\sum_{\gamma=1}^{\nu} K_{\gamma}\left(\frac{\partial \mu_{\alpha}}{\partial n_{\gamma}}\right)_{T, n_{\bar{\gamma}}}, \\
\phi_{\mathrm{JJ}}=\frac{1}{\rho} L_{\mathrm{JJ}}, & \phi_{\mathrm{HH}}=\frac{1}{T \mathrm{C}_{\mathrm{V}}} L_{\mathrm{HH}} .
\end{array}
$$

Thereafter, we have all the needed quantities for the subsequent calculations of collective mode spectrum in the hydrodynamic limit.

\footnotetext{
${ }^{1}$ It is worth noting that in contrary to the definitions for the thermal conductivity considered within phenomenological theories (see, e.g., [12]) this definition takes into account partialy the diffusion fluxes $I_{\alpha}^{d}($ see $(85))$.
} 


\section{Spectrum of hydrodynamic collective modes for a multi- component fluid}

\subsection{General, chemically nonreactive mixture}

The hydrodynamic collective modes can be found as the solution of the equation

$$
\operatorname{det}\left[\begin{array}{ccc}
z \delta_{\alpha \beta}+k^{2} \phi_{\mathrm{NN}}^{\alpha \beta} & -\frac{i k}{\bar{m}} c_{\alpha} & k^{2} \phi_{\mathrm{NH}}^{\alpha} \\
-\frac{i k}{\kappa_{\mathrm{T}}} v_{\alpha} & z+k^{2} \phi_{\mathrm{JJ}} & -\frac{i k}{\mathrm{C}_{\mathrm{V}}} \frac{\alpha_{\mathrm{P}}}{k_{\mathrm{T}}} \\
k^{2} \phi_{\mathrm{HN}}^{\alpha} & -\frac{i k T}{\rho} \frac{\alpha_{\mathrm{P}}}{k_{\mathrm{T}}} & z+k^{2} \phi_{\mathrm{HH}}
\end{array}\right]=0,
$$

where $\alpha, \beta$ are from the set $1,2, \ldots, \nu$. The equation $(94)$ is of $(\nu+2)$ th order with respect to $z$. Looking for the solutions in the form

$$
z=z_{0} k+z_{1} k^{2}+\cdots,
$$

the coefficients $z_{0}$ and $z_{1}$ may be calculated.

After some mathematical manipulations it can be shown that the equation (94) has in general: two complex-conjugated solutions in the form

$$
z_{s}= \pm c_{s} k-D_{s} k^{2}
$$

and $\nu$ purely real solutions describing a heat mode with

$$
z_{h}=-\Gamma k^{2}
$$

as well as $\nu-1$ diffusion modes with

$$
z_{d}^{r}=-\mathcal{D}_{r} k^{2}, \quad r=\{1,2, \ldots, \nu-1\},
$$

where $\Gamma$ and $\mathcal{D}_{r}$ are the damping coefficients of the heat and diffusion modes, respectively. One can show that the sound velocity $c_{s}$ is given by (78) as it was expected. Using the structure of (94) we found also the expression for the sound damping coefficient $D_{s}$ in the form

$$
D_{s}=\frac{\eta_{l}}{2 \rho}+\frac{\gamma-1}{2 \mathrm{C}_{\mathrm{P}}} \lambda+\frac{1}{2 \gamma k_{\mathrm{T}}}\left(\mathbf{v}^{+} \mathbf{D} \mathbf{v}\right)+\frac{\alpha_{\mathrm{P}}}{\mathrm{C}_{\mathrm{P}} k_{\mathrm{T}}}\left(\mathbf{v}^{+} \mathbf{K}\right),
$$

where $\mathbf{D}$ and $\mathbf{K}$ are the matrix and the vector-column with the elements $D_{\alpha \beta}$ and $K_{\alpha}$, respectively. The result (99) agrees with the expression found previously by phenomenological theories $[13,20]$

To calculate the diffusion damping coefficients $\mathcal{D}_{r}$, where $r=\{1, \ldots, \nu-$ $1\}$, one has to consider each from the cases with fixed value of $\nu$ separately. We will study below as an example a binary mixture of simple fluids with $\nu=2$.

For transverse components one has the purely real mode

$$
z_{t}=-\frac{\eta}{\rho} k^{2},
$$

similar as it is for a simple fluid. 


\subsection{Hydrodynamic modes for a binary mixture}

Taking into account the relations (91) and (91), the normalized diffusion D and thermal diffusion $\mathrm{K}$ coefficients can be defined as follows

$$
D_{\alpha \beta}=(-1)^{\alpha+\beta} \frac{\mathrm{D}}{m_{\alpha} m_{\beta}}, \quad K_{\alpha}=(-1)^{\alpha+1} \frac{\mathrm{K}}{m_{\alpha}}
$$

where $\alpha, \beta=1,2$. Thus, for elements of the matrix of memory functions one has

$$
\begin{aligned}
\phi_{\mathrm{NN}}^{11}=\mathrm{D} \frac{g_{1}}{m_{1}}, & \phi_{\mathrm{NN}}^{12}=-\mathrm{D} \frac{g_{2}}{m_{1}}, \\
\phi_{\mathrm{NN}}^{21}=-\mathrm{D} \frac{g_{1}}{m_{2}}, & \phi_{\mathrm{NN}}^{22}=\mathrm{D} \frac{g_{2}}{m_{2}}, \\
\phi_{\mathrm{HN}}^{1}=\mathrm{K} g_{1}, & \phi_{\mathrm{HN}}^{2}=-K g_{2},
\end{aligned}
$$

and

$$
\phi_{\mathrm{NH}}^{1}=\frac{1}{T \mathrm{C}_{\mathrm{v}} m_{1}} \mathrm{~K}, \quad \phi_{\mathrm{NH}}^{2}=-\frac{1}{T \mathrm{C}_{\mathrm{v}} m_{2}} \mathrm{~K},
$$

where

$$
\begin{gathered}
g_{1}=\frac{1}{m_{1}}\left(\frac{\partial \mu_{1}}{\partial n_{1}}\right)_{V, T, n_{2}}-\frac{1}{m_{2}}\left(\frac{\partial \mu_{1}}{\partial n_{2}}\right)_{V, T, n_{1}}=\frac{1}{\rho}\left(\frac{\partial \mu_{1}}{\partial x_{1}}\right)_{V, T, M}, \\
g_{2}=-\frac{1}{m_{1}}\left(\frac{\partial \mu_{2}}{\partial n_{1}}\right)_{V, T, n_{2}}+\frac{1}{m_{2}}\left(\frac{\partial \mu_{2}}{\partial n_{2}}\right)_{V, T, n_{1}}=-\frac{1}{\rho}\left(\frac{\partial \mu_{2}}{\partial x_{1}}\right)_{V, T, M} .
\end{gathered}
$$

Thereafter, for a binary mixture the equation (94) can be rewritten in the form

$$
\operatorname{det}\left[\begin{array}{cccc}
z+k^{2} \mathrm{D} \frac{g_{1}}{m_{1}} & -k^{2} \mathrm{D} \frac{g_{2}}{m_{1}} & -\frac{i k}{\bar{m}} c_{1} & k^{2} \frac{\mathrm{K}}{T \mathrm{C}_{\mathrm{v}} m_{1}} \\
-k^{2} \mathrm{D} \frac{g_{1}}{m_{2}} & z+k^{2} \mathrm{D} \frac{g_{2}}{m_{2}} & -\frac{i k}{\bar{m}} c_{2} & -k^{2} \frac{\mathrm{K}}{T \mathrm{C}_{\mathrm{v}} m_{2}} \\
-\frac{i k}{\kappa_{\mathrm{T}}} v_{1} & -\frac{i k}{\kappa_{\mathrm{T}}} v_{2} & z+k^{2} \frac{\eta_{l}}{\rho} & -i k \frac{\alpha_{\mathrm{P}}}{\mathrm{C}_{\mathrm{v}} \kappa_{\mathrm{T}}} \\
k^{2} \mathrm{~K} g_{1} & -k^{2} \mathrm{~K} g_{2} & -i k \frac{T \alpha_{\mathrm{P}}}{\rho \kappa_{\mathrm{T}}} & z+k^{2} \frac{\lambda}{\mathrm{C}_{\mathrm{V}}}
\end{array}\right]=0 .
$$

Let us solve the equation (107) looking for the solutions in the form

$$
z=z_{0} k+z_{1} k^{2}+\ldots
$$

Thus, we get:

a) two complex-conjugated sound modes with

$$
z_{s}= \pm i c_{s} k-D_{s} k^{2}
$$


where the sound velocity $c_{s}$ is given by the expression (78) and the damping coefficient $D_{s}$ may be written as follows

$$
2 D_{s}=\frac{\eta_{l}}{\rho}+\frac{(\gamma-1)}{\mathrm{C}_{\mathrm{P}}} \lambda+\frac{2 \alpha_{P} \bar{\delta}}{\kappa_{\mathrm{T}} \mathrm{C}_{\mathrm{P}}} \mathrm{K}+\frac{\bar{\delta}^{2}}{\gamma \kappa_{\mathrm{T}}} \mathrm{D} .
$$

This result is in agreement with (99). Note that

$$
\bar{\delta}=\frac{v_{1}}{m_{1}}-\frac{v_{2}}{m_{2}}=\frac{1}{\rho} \delta_{x},
$$

where $x \equiv x_{1}$ and

$$
\delta_{x}=\frac{1}{V}\left(\frac{\partial V}{\partial x}\right)_{P, T, M}
$$

is so-called ' $N-x$ ' dilatation [22,24].

b) a hydrodynamic heat mode with

$$
z_{h}=-\Gamma k^{2}, \quad \Gamma=\frac{1}{2}\left[a_{1}+\sqrt{a_{1}^{2}-4 a_{0}}\right],
$$

where

$$
\begin{gathered}
a_{1}=\frac{\lambda}{\mathrm{C}_{\mathrm{P}}}-2 \frac{\alpha_{\mathrm{P}} \bar{\delta}}{\kappa_{\mathrm{T}} \mathrm{C}_{\mathrm{P}}} \mathrm{K}+\mathrm{D}\left[\frac{z_{x}}{\rho}-\frac{1}{\gamma \kappa_{\mathrm{T}}} \bar{\delta}^{2}\right], \\
a_{0}=\frac{1}{T \mathrm{C}_{\mathrm{P}}}\left[\frac{z_{x}}{\rho}-\frac{1}{\kappa_{\mathrm{T}}} \bar{\delta}^{2}\right]\left(\mathrm{D} \lambda T-\mathrm{K}^{2}\right),
\end{gathered}
$$

and

$$
z_{x}=\left(\frac{\partial \mu_{x}}{\partial x}\right)_{V, T, M}, \quad \mu_{x}=\frac{\mu_{1}}{m_{1}}-\frac{\mu_{2}}{m_{2}} .
$$

c) a hydrodynamic diffusion mode with

$$
z_{d}=-\mathcal{D} k^{2}, \quad \mathcal{D}=\frac{1}{2}\left[a_{1}-\sqrt{a_{1}^{2}-4 a_{0}}\right],
$$

and the coefficients $a_{1}$ and $a_{0}$ are defined by equations (112)-(113).

It can be shown that the obtained results (110), (111) and (115) agree with the results known previously from phenomenological treatment [23, $22,13]$. However, the damping coefficients are written in forms which differ from most commonly quoted because of using different definitions of phenomenological coefficients (see also [21]).

\section{Conclusion}

We have applied the scheme of the generalized hydrodynamics to a multicomponent, nonreacting fluid for deriving the basic equations of generalized hydrodynamics. This makes possible to use the generalized mode approach developed previously for a simple fluid for the subsequent study of timecorrelation functions, generalized collective mode spectrum and generalized transport coefficients of a mixture.

We have demonstrated one to one correspondence between the results for hydrodynamic collective modes obtained from the equations of generalized hydrodynamics and those found by phenomenological theories. It is worth to note also that the expressions for the generalized $k$-dependent thermodynamic quantities of a mixture have been derived rigorously for the first time herein. 


\section{References}

[1] Kamgar-Parsi B., Cohen E.G.D., de Schepper I.M. Dynamic processes in hardsphere fluids. // Phys. Rev. A, 1987, vol. 35, No 11, p. 4781-4795.

[2] De Schepper I.M., Cohen E.G.D., Bruin C., van Rijs J.C., Montrooij W., de Graaf L.A. Hydrodynamic time correlation functions for Lennard-Jones fluids. // Phys. Rev. A, 1988, vol. 38, No 1, p. 271-287.

[3] Mryglod I.M., Omelyan I.P., Tokarchuk M.V. Generalized collective modes for the Lennard-Jones fluid. // Mol. Phys., 1995, vol. 84, No 2, p. 235-259.

[4] Omelyan I.P., Mryglod I.M. Generalized collective modes of a Lennard-Jones fluid. High-mode approximation. // Cond. Matt. Phys., 1994, vol. 4, p. 128160.

[5] Mryglod I.M., Omelyan I.P. Generalized collective modes for a Lennard-Jones fluid in higher mode approximations. // Phys. Lett. A, 1995, vol. 205, p. 401406.

[6] Mryglod I.M., Omelyan I.P. Generalized mode approach: I. Transverse time correlation functions and generalized shear viscosity of a Lennard-Jones fluid. // Mol. Phys., 1997 (in press).

[7] Miura S. Anomalous acoustic dynamics in liquid water: the origin of low frequency mode. // Mol. Phys., 1996, vol. 87, No 6, p. 1405-1421.

[8] Bosse J., Jacucci G., Ronchetti M., Schirmacher W. Fast sound in twocomponent liquids. // Phys. Rev. Lett., 1986, vol. 57, No 26, p. 3277-3279.

[9] Westerhuijs P., Montfrooij W., de Graaf L.A., de Schepper I.M. Fast and slow sound in a dense gas mixture of helium and neon. // Phys. Rev. A, 1992, vol. 45 , No 6, p. 3749-3762.

[10] Smorenburg H.E., Crevecoeur R.M., de Schepper I.M. Fast sound in a dense helium argon gas mixture. // Phys. Lett. A, 1996, vol. 211, p. 118-124.

[11] Smorenburg H. Microscopic dynamics of binary mixtures and quasi-colloidal systems. Ph.D. Thesis, TU Delft (1996), 97p.

[12] De Groot S.P., Mazur P. Non-equilibrium thermodynamics. New York, Dover Publ., 1984.

[13] Landau L.D., Lifschitz E.M. Fluid mechanics. London, Pergamon Press, 1955.

[14] Boley C.D., Yip S. Kinetic theory of time-correlation functions in a binary gas mixture. // Phys. Fluids, 1972, vol. 15, No 8, p. 1433-1446.

[15] Lorez de Haro M., Cohen E.G.D. The Enskog theory for multicomponent mixtures. III. Transport properties of dense binary mixtures with one tracer component. // J. Chem. Phys., 1984, vol. 80, No 1, p. 408-415.

[16] Zubarev D.N. Modern methods of the statistical theory of nonequilibrium processes. In: Itogi Nauki i Tekhniki, Sovremennyje Problemy Matematiki. VINITI, 1980, vol. 15, p. 131-226 (in Russian).

[17] Mryglod I.M., Hachkevych A.M. On non-equilibrium statistical theory of a fluid: Linear relaxation theory. // Cond. Matt. Phys., 1995, vol. 5, p. 105118.

[18] Boon J.P., Yip S. Molecular hydrodynamics. New York, McGraw-Hill, 1980.

[19] Hansen J.-P., McDonald I. R. Theory of simple liquids. London, Academic Press, 1986.

[20] Jordan P.C., Jordan J.R. Nonequilibrium statistical mechanics of a multicomponent fluid. // J. Chem. Phys., 1966, vol. 45, No 7, p. 2492-2507.

[21] Mryglod I.M., Ignatyuk V.V. Generalized hydrodynamics of binary mixtures. // J. Phys. Studies, 1997 (submitted).

[22] Bhatia A.B., Thornton D.E., March N.H. Dynamic structure factors for a fluid binary mixture in the hydrodynamic limit. // Phys. Chem. Liq., 1974, vol. 4, p. 97-111.

[23] Mountain R.D., Deutch J.M. Light scattering from binary solutions. // J. Chem. Phys., 1969, vol. 50, No 3, p. 1103-1108.

[24] March N.M., Tosi M.P. Atomic dynamics in liquids. London, Macmillan, 1976.

[25] Caccamo C. Integral equation theory description of phase equilibria in classical fluids. // Phys. Rep., 1996, vol. 274, No 1/2, p. 1-105. 


\title{
УЗАГАЛЬНЕНА ГІДРОДИНАМІКА
}

БАГАТОКОМПОНЕНТНИХ РІДИН

\begin{abstract}
I.М.Мриглод
На основі строгого мікроскопічного розгляду, приймаючи до уваги усі величини, що зберігаються, отримано базові рівняння узагальненої гідродинаміки багатокомпонентної, хімічно нереагуючої рідини. Записані мікроскопічні вирази для коефіцієнтів переносу, розраховано коефіцієнти затухання та дисперсія гідродинамічних колективних збуджень системи. Показано, що отримані результати узгоджуються з розрахунками, які виконані на основі феноменологічних теорій.
\end{abstract}

